\title{
Laboreal
}

Volume $17 \mathrm{~N}^{\circ} 1$ | 2021

Trabalhar hoje: mudanças, permanências, estratégias, reinvenções

\section{Trabajar hoy: cambios, permanencias, estrategias, reinvenciones - presentación del Dosier}

Trabalhar hoje: mudanças, permanências, estratégias, reinvenções -

apresentação do Dossiê

Travailler aujourd'hui: changements, permanences, stratégies, réinventions -

présentation du Dossier

Working today: changes, permanencies, strategies, reinventions - Dossier

presentation

Anísio José da Silva Araújo, Mary Yale Neves, Manuella Castelo Branco Pessoa y Thaís Augusta Maximo

Traductor. Fernanda Romero (fernandaromero.trad@gmail.com)

\section{(2) OpenEdition}

\section{Journals}

Edición electrónica

URL: https://journals.openedition.org/laboreal/17664

DOI: $10.4000 /$ laboreal. 17664

ISSN: 1646-5237

Editor

Universidade do Porto

Referencia electrónica

Anísio José da Silva Araújo, Mary Yale Neves, Manuella Castelo Branco Pessoa y Thaís Augusta Maximo, «Trabajar hoy: cambios, permanencias, estrategias, reinvenciones - presentación del Dosier», Laboreal [En línea], Volume 17 NN$^{0} 1$ | 2021, Publicado el 18 junio 2021, consultado el 20 junio 2021 URL: http://journals.openedition.org/laboreal/17664 ; DOI: https://doi.org/10.4000/laboreal.17664

Este documento fue generado automáticamente el 20 junio 2021.

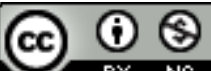

Laboreal está licenciado com uma Licença Creative Commons - Atribuição-NãoComercial 4.0 Internacional. 


\title{
Trabajar hoy: cambios, permanencias, estrategias, reinvenciones - presentación del Dosier
}

\author{
Trabalhar hoje: mudanças, permanências, estratégias, reinvenções - \\ apresentação do Dossîe \\ Travailler aujourd'hui: changements, permanences, stratégies, réinventions - \\ présentation du Dossier \\ Working today: changes, permanencies, strategies, reinventions - Dossier \\ presentation
}

Anísio José da Silva Araújo, Mary Yale Neves, Manuella Castelo Branco Pessoa y Thaís Augusta Maximo

Tradución : Fernanda Romero (fernandaromero.trad@gmail.com)

\section{El trabajo se modifica}

1 El trabajo se modifica. Schwartz y Durrive (2010) parten de esta premisa al afirmar que, además de las transformaciones substantivas, radicales, que marcan ciertos momentos de la historia de los mundos del trabajo, en los cuales estas se presentan incontestables y especialmente evidentes, el trabajo no cesa su transmutación. La comprobación de esa modificación constante ocurre por diferentes vías, entre las que se destacan la sociología y la del punto de vista de la actividad.

2 Por la vía sociológica (Antunes, 2020; Boltanski \& Chiapello, 2009; Linhart, 2017) se captan las mutaciones en sus caracteres generales, en sus tendencias, en sus orientaciones. Por otro lado, por la vía de la actividad (Wisner, 1994; Daniellou, 2004), se ve con especial claridad no sólo cómo se modifica el trabajo, sino cómo se manifiestan y procesan estas remodelaciones por parte de los protagonistas del trabajo. 
En otros términos, cómo estas inciden en la vida, en la salud, en la subjetividad y en las actividades de los trabajadores y trabajadoras y, además, qué reacciones, acciones, retrocesos, enfrentamientos, miedos y angustias, suscitan.

Privilegiada por Laboreal, la vía del curso de la actividad actúa, entonces, como un antídoto contra eventuales intentos de ciertos extractos de la comunidad científica de deducir el particular de lo general, dispensándose de hacer un movimiento en la dirección de dar voz a quienes vivencian las modificaciones en su hacer. Por ese ángulo, se trata de una búsqueda de encarnar el cambio, de percibirlo en la intimidad de quién trabaja, en su permanente esfuerzo por afirmarse como humano, que produce nuevas normas de vida, que desea transformar su medio en algo visible (Schwartz, 2000, 2010), a pesar de todo.

4 Este Dosier ha pretendido dirigir la mirada hacia el trabajo en la actualidad. Fuente de perplejidad, de recelos, de tenebrosas proyecciones y de gran angustia y, en simultaneo, generador de un impulso ineludible para conocer mejor lo que ocurre en él y que contornos el cambio presagiado asume, en el inicio, como siendo de la naturaleza del trabajo.

\section{Cambios, permanencias, estrategias y reinvenciones en los mundos del trabajo}

Han sido varios los motivos que orientaron la adopción de este tema. En primer lugar, se pude destacar el surgimiento de distintas ocupaciones procedentes de la intensificación del uso de las nuevas tecnologías. Partiendo de esta perspectiva, han ido creciendo los estudios para mapear y caracterizar estas nuevas ocupaciones, específicamente en lo que respecta a una de las formas de trabajo que viene ganando evidencia en el trabajo actual: el trabajo "uberizado" o basado en aplicaciones (Antunes, 2020; Abílio, 2020; Bridi \& Lima, 2018; Linhart, 2017; Slee, 2017). Dichos autores aportan también un importante análisis sobre la pérdida de derechos laborales y de seguridad social con la informalización del trabajo, producto, de entre otros factores, de estas nuevas formas de trabajo, dicho "autónomas".

6 Además, es apremiante comprender las nuevas características que adquiere el llamado trabajo regulado. Este no ha estado paralizado en el tiempo, pero se ha modificado en función de las presiones ejercidas por las inflexiones económicas, políticas y sociales. Se hizo necesario dimensionar estas modificaciones, comprendiendo no solo las implicaciones que ejercen en los trabajadores y trabajadoras, sino también en aquellos que están al margen de lo regulado, buscando formas diferentes de sobrevivir y existir en la sociedad. Las presiones nacionales e internacionales van en el sentido de flexibilizar cada vez más el trabajo regulado, resultando en pérdidas laborales, desprotección social y aumento exponencial de la informalidad. Se percibe, así, una tendencia creciente hacia la desregulación, en el sentido de la liberación de cualquier vínculo contractual o jurídico, acercándose al destino de las nuevas ocupaciones profesionales del trabajo a través de aplicaciones.

7 Si bien siempre estuvo presente en el paisaje latinoamericano, especialmente en el brasileño, el trabajo informal asume dimensiones jamás vistas e incluso es escandalosamente propuesto como el nuevo paradigma del trabajo, la encarnación de la utopía capitalista capaz de recorrer su camino de explotación sin obstáculos. Así 
mismo, el avance de la precarización del trabajo, impulsado por las modificaciones de la legislación laboral que colocan a los trabajadores y trabajadoras en una posición de creciente fragilidad, situación agravada por las altas tasas de desempleo que, por su vez, inhibe y sofoca muchas de sus reacciones en el sentido de la defensa y profundización de las conquistas obtenidas.

Hay también que registrar el empeoramiento de los indicadores de cansancio, desgaste, sufrimiento y de dolencia -sobre todo a nivel psíquico- relacionados con el trabajo en la actualidad (Han, 2021; Dejours, 2004, 2012; Seligmann-Silva, 2011). Esta realidad parece ser más evidente para quienes desarrollan sus actividades sometidos a una explotación desmedida y sin ninguna protección laboral y de seguridad social, o por la falta de acceso al trabajo remunerado (Seligmann-Silva, 2011). A esto hay que sumar el desmoronamiento de los colectivos del trabajo y el desmantelamiento de la actividad sindical, que ha ido perdiendo fuerzas en el transcurso del proceso de desarticulación de la sociedad regulada o salarial, condenando muchas categorías profesionales al aislamiento frente a los embates del capital.

Dichas constataciones constituyen la matriz de una gran consternación porque, como afirmado en la introducción de este Dosier, a lo que se asiste nos remite a un pasado de sobreexplotación y ausencia de derechos que se imaginaba en vías de ser suplantado. Incluso en el marco del capitalismo, se creía posible contener su desenfrenado impulso de acumulación por la vía del control social, lo que no ocurrió. De hecho, la ideología neoliberal se propagó e invadió, sin escrúpulos, el discurso de gobernantes y de empresas, en un contexto de ascensión de los proyectos ultraconservadores. Tal contratiempo fue también una de las causas del tema en revisión, porque no sólo hay un deseo de conocer, sino también, en la medida de lo posible, de aportar elementos que puedan redireccionar el curso de los acontecimientos, apuntando el trabajo hacia nuevos horizontes.

10 Así, pensando desde antes de la grave crisis sanitaria del COVID-19 que asola al mundo, el propósito de este número de Laboreal fue observar de cerca algunas de estas modificaciones, situándose en el radio de la actividad, comprendiendo, más allá de los efectos que producen en las condiciones de vida y de trabajo, qué formas de resistencia y apropiación de la actividad engendran. Buscamos, de ese modo, circular entre el macro y el micro, privilegiando el análisis de situaciones reales de trabajo. Es esa "mirada con lupa", tal como sugiere Schwartz (2003, 2010), la que nos permitirá comprender por dentro las dimensiones objetivas y subjetivas de las mutaciones en curso, permitiéndonos ofrecer nuevas perspectivas para los fenómenos investigados.

11 De esta perspectiva asumida procede una exigencia: el rigor metodológico, que busca incansablemente las vías posibles y pertinentes para captar este cambio en proceso, en el diálogo entre investigadores y trabajadores y trabajadoras. Este fue el reto permanente de los editores y organizadores, especialmente en tiempos de pandemia, donde se reconfiguran tantas situaciones, sobre todo en los mundos del trabajo y de la economía, golpeados duramente por esta realidad.

12 En lo que respecta al escenario pandémico actual, se constata un loable empeño por parte de los investigadores para otorgar visibilidad, por ejemplo, a la intensificación de las desigualdades, incluidas las que remiten a la división sexual del trabajo (Hirata \& Kergoat, 2007; Hirata \& Zarifian, 2009). Hay, por lo tanto, un amplio espectro de cambios, cuya aprehensión está lejos de considerarse satisfactoria. A su vez, el distanciamiento social preconizado por las autoridades sanitarias impuso grandes 
dificultades a la investigación, pese a que estimuló la exploración de nuevas posibilidades que pudiesen preservar la aprehensión diligente desde el punto de vista de la actividad.

\section{El trabajo hoy y las aportaciones presentadas en este Dosier}

13 Algunas iniciativas de investigación aquí documentadas han caminado en esa dirección. Los artículos publicados buscan retratar la complejidad que representa trabajar en el presente, en sus cambios y permanencias. Por ello, el Dosier contempla cuatro estudios, dos de los cuales discuten la "uberización" del trabajo y la radiodifusión, y otros dos que buscan desvelar, ya sea la pandemia de COVID-19 en su relación con el trabajo doméstico de las madres o el trabajo de los profesionales de la salud que están en contacto directo y constante con el virus, sometidos a un mayor riesgo de contagio debido a la naturaleza de la función que desempeñan.

El artículo, que tiene como autores a Wladimir Ferreira de Souza y Milton Athayde, titulado "Dinámica psicológica y trabajo de los profesionales de la salud en Brasil durante la pandemia de COVID-19: contribuyendo a comprender $\leftrightarrow$ transformar su experiencia", presenta un importante debate en torno a cuestiones relativas al trabajo en el área de la salud en Brasil, con el contexto pandémico como telón de fondo. Para ello, se utilizan del punto de vista de la actividad a partir de referencias de la Ergonomía de la Actividad, de la Ergología (Schwartz, 2010) y de otros enfoques clínicos del trabajo (Clot, 2011; Clot \& Lhuilier, 2013; Dejours, 2004, 2012).

El texto está organizado de manera a situar el marco del COVID-19 como emergencia internacional, mencionando las características generales de las situaciones de trabajo que vivencian los profesionales de la salud. En este seguimiento, los autores presentan informes de experiencias procedentes de una asociación desarrollada entre la Universidad del Estado de Río de Janeiro (UERJ) y el Centro de Referencia en Salud del Trabajador (CEREST). Sobresalen algunos aspectos: la presencia preponderante de mujeres entre los profesionales de la salud, lo que nos remite a los dilemas y la sobrecarga inherentes al conflicto/conciliación entre trabajo productivo/trabajo reproductivo. Otro aspecto se refiere a la importancia de la dimensión colectiva del trabajo, reforzando la esencialidad de los colectivos para trabajar en la salud.

En el artículo "Un análisis de las normas antecedentes y reservas de alternativas movilizadas en la actividad de los conductores y repartidores a través de aplicaciones", las autoras -Denise Alvarez, Letícia Pessoa Masson, Cirlene Christo, Samara Leal, Gabriela Siqueira Salomão y Sarah de Paulo do Amaral- discuten, desde la perspectiva ergológica (Schwartz \& Durrive, 2010), las normas antecedentes que inciden en las actividades de estos trabajadores en la ciudad de Río de Janeiro, con el fin de abordar las implicaciones de estas formas actuales de control y gestión algorítmica del trabajo. Así mismo, buscan identificar las especificidades allí presentes, bien como examinar los elementos del hacer colectivo que remiten a la movilización y a la construcción de reservas de alternativas (Schwartz, 2010) por los conductores y repartidores, en un escenario anterior y posterior a la pandemia de COVID-19.

Los análisis realizados en ese estudio ponen de manifiesto las exigencias y los retos inherentes a este modo de gestión, así como las renormatizaciones efectivadas en la 
construcción de reservas de alternativas (Schwartz, 2010), a ejemplo de la formación de redes de sociabilidad de apoyo en las redes sociales, que surgen como instrumento de difusión de información y de ayuda, sustituyendo, en cierta medida, la asistencia que deberían prestar las empresas. Asimismo, se discute la ausencia de regulación estatal y el modus operandi de las empresas de plataforma, cuya sofisticada y compleja tecnología contribuye a una nueva etapa de profundización del proceso de precarización social y de explotación de los trabajadores, resultante de una verdadera subsunción del trabajador por la moderna capacidad de gestionar el trabajo en remoto, a tiempo completo (Abilio, 2020; Linhart, 2017).

En el artículo "El trabajo doméstico no remunerado de las madres en la pandemia de COVID-19: cambios y permanencias", la autora Lívia Borges Hoffmann Dorna, ofrece a los lectores discusiones sobre los cambios y permanencias en el trabajo doméstico no remunerado, centrándose en la cuestión del cuidado del hogar y de los niños durante la pandemia de COVID-19. Las cuestiones de género y trabajo, en el contexto de la crisis sanitaria, han adquirido tal visibilidad que la Organización de las Naciones Unidas (ONU Mujeres, 2020) ha emitido una alerta mundial en la que se destacan los impactos de las emergencias de salud pública desde una perspectiva de género.

Basándose en el marco teórico de la Ergología (Schwartz \& Durrive, 2010), la articulista resalta la urgencia de ampliar el concepto de trabajo, haciendo referencia a la división social y sexual del trabajo (Kergoat, 2009; Hirata \& Kergoat, 2007). La investigación se realizó a partir de una encuesta en línea con 360 madres brasileñas con hijos en la franja de edad de cero a 12 años. Los resultados demostraron la desigual distribución del trabajo reproductivo entre los sexos, revelando un aumento significativo de las horas dedicadas por las mujeres a estas tareas. Se identificaron varias quejas relacionadas con la sobrecarga física y mental, además de las dificultades específicas derivadas del aislamiento social. Asimismo, algunas familias han ideado nuevos acuerdos de distribución de tareas, haciendo visible la crucialidad y la penosidad del trabajo doméstico.

El artículo titulado "Ergología y radiodifusión: análisis del trabajo de mantenimiento y soporte en televisión", de la autoría de Raquel Figueira Lopes Cancado-Andrade y Alexandre de Carvalho Castro, tuvo como objetivo analizar la actividad de los trabajadores de mantenimiento y soporte (MeS), responsables de apoyar la producción y transmisión en la radiodifusión en Brasil. Este sector implica la producción y difusión de contenidos periodísticos, educativos y de entretenimiento a gran escala. Los autores fundamentan sus análisis desde la perspectiva ergológica (Schwartz \& Durrive, 2010) y bajtiniana (Bajtín, 1952/2003), destacando, a partir de los resultados encontrados, que esta actividad es comúnmente referida bajo el signo de la "técnica", un eslogan reduccionista que no adjetiva una acción, sino que encubre la necesidad de una formación especializada por parte del trabajador, que se encuentra en el papel de ser cobrado por su acto.

Dicho hábito confiere un carácter de impersonalidad a los trabajadores de MeS, "sin rostro, sin nombre, sin reconocimiento". La cuestión de la disponibilidad total también gana destaque en los análisis realizados. A partir de las normas antecedentes y de los valores del grupo, los autores señalan que los trabajadores son cobrados en su totalidad en esta organización productiva. El estrés y el ajetreo se enfrentan en función de la renormalización realizada por los trabajadores en una actividad marcada por la 
imprevisibilidad, en una considerable asimetría entre el trabajo prescrito y el trabajo real.

En general, la actividad de los trabajadores de mantenimiento y soporte está atravesada por disputas entre los equipos de trabajo, sin contar con la presión permanente por la plena disponibilidad y la exigencia de resultados inmediatos. Los autores enfatizan cómo la dinámica de trabajo en la radiodifusión es susceptible a los cambios sociales, a las impermanencias y a las reinvenciones productivas, una vez que están marcadamente influenciadas por las innovadoras producciones de contenidos.

\section{A modo de conclusión}

Esta breve e incompleta presentación traduce el alcance de los cambios y permanencias vivenciados en este tiempo. Los acontecimientos en el mundo del trabajo están repletos de nuevos retos, que exigen un esfuerzo para aprehender las distintas modificaciones en profundidad, desde distintos ángulos, estructurando análisis que reflejen la complejidad en curso. En lo que respecta a este número temático, las "provocaciones" no se encierran en él, sino que permanecen como una invitación al desarrollo investigativo y diligente con miras a reducir, a través del conocimiento, la perplejidad siempre presente en el origen de la ignorancia. Así, además de contribuir a la comprensión de lo que ocurre alrededor, se espera fomentar transformaciones en las condiciones de vida, salud y trabajo.

\section{BIBLIOGRAFÍA}

Abílio, L. (2020). Uberização: a era do trabalhador just-in-time? Estudos Avançados, 34(98), 111-126. https://doi.org/10.1590/s0103-4014.2020.3498.008

Antunes, R. (2020). Uberização, trabalho digital e indústria 4.0. São Paulo: Boitempo.

Bakhtin, M. (1952/2003). Os gêneros do discurso. In M. Bakhtin (Ed.), Estética da criação verbal (pp. 261-306). Organização, tradução e nota Paulo Bezerra. São Paulo: Martins Fontes.

Boltanski, L., \& Chiapello, È. (2009). O novo espírito do capitalismo. São Paulo: Editora WMF Martins Fontes.

Clot, Y. (2010). Trabalho e poder de agir. Belo Horizonte: Fabrefactum.

Clot, Y., \& Lhuilier, D. (2013). Agir en clinique du travail. Paris: Érès.

Daniellou, F. (2004). A Ergonomia em busca de seus princípios: debates epistemológicos. São Paulo: Edgard Blucher.

Dejours, C. (2004). Subjetividade, trabalho e ação. Produção, 14(3), 27-34. https://doi.org/10.1590/ S0103-65132004000300004

Dejours, C. (2012). Trabalho vivo. Trabalho e emancipação. Brasília: Paralelo 15. 
Haicault, M. (2020). La charge mentale. Histoire d'une notion charnière (1976-2020). Retirado de https://hal.archives-ouvertes.fr/hal-02881589

Han, B. C. (2015). Sociedade do cansaço. Petrópolis: Vozes.

Hirata, H., \& Kergoat, D. (2007). Novas configurações da divisão sexual do trabalho. Cadernos de Pesquisa, 37(132), 595-609. https://doi.org/10.1590/S0100-15742007000300005

Hirata, H., \& Zarifian, P. (2009). Trabalho (o conceito de). In H. Hirata, F. Laborie, H. Le Doaré, \& D. Senotier (Orgs.), Dicionário Crítico do Feminismo (pp. 251-255). São Paulo: Editora Unesp.

Kergoat, D. (2009). Divisão sexual do trabalho e relações sociais de sexo. In H. Hirata, F. Laborie, H. Le Doaré, \& D. Senotier (Orgs.), Dicionário Crítico do Feminismo (pp. 67-75). São Paulo: Unesp.

Lima, J. C., \& Bridi, M. A. (2018). Flexíveis, virtuais e precários? Os trabalhadores em tecnologia da informação. Curitiba: Editora UFPR.

Linhart, D. (2017, 01 de março de 2021). A uberização do trabalho não é inevitável. Um assalariado sem submissão é possível. Le Monde Diplomatique Brasil, Edição - 120. Retirado de https:// diplomatique.org.br/um-assalariado-sem-submissao-e-possivel/

Schwartz, Y. (2000). Le paradigme ergologique ou um métier de philosophe. Toulouse: Octares.

Schwartz, Y., \& Durrive, L. (2010). Trabalho \& ergologia: conversas sobre a atividade humana (2 edição). Niterói: EdUFF.

Seligmann-Silva, E. (2011). Trabalho e desgaste mental: o direito de ser dono de si mesmo. São Paulo: Cortez.

Slee, T. (2017). Uberização: a nova onda do trabalho precarizado. São Paulo: Elefante.

Wisner, A. (1994). A inteligência do trabalho: textos selecionados de ergonomia. São Paulo: Fundacentro.

\section{AUTORES}

\section{ANÍSIO JOSÉ DA SILVA ARAÚJO}

https://orcid.org/0000-0002-3128-3897

Universidade Federal da Paraíba, Cidade Universitária, João Pessoa, PB - Brasil, CEP: 58051-900 anisiojsa@uol.com.br

\section{MARY YALE NEVES}

https://orcid.org/0000-0002-9821-3826

Universidade Federal Fluminense, Rua Clarice Índio do Brasil, 30/803, Botafogo, Rio de Janeiro, Brasil, CEP: 22.230-090

myale@uol.com.br

\section{MANUELLA CASTELO BRANCO PESSOA}

https://orcid.org/0000-0003-3523-8708

Universidade Federal da Paraíba, Cidade Universitária - João Pessoa, PB - Brasil, CEP: 58051-900

manucastelobranco2@gmail.com 


\section{THAÍS AUGUSTA MAXIMO}

https://orcid.org/0000-0002-5062-1548

Universidade Federal da Paraíba, Cidade Universitária - João Pessoa, PB - Brasil, CEP: 58051-900

thais.maximo@academico.ufpb.br 